\title{
Christentum und Judentum in Schleiermachers Vorlesungen über
} die Kirchengeschichte

In:

Christentum und Judentum : Akten des Internationalen Kongresses der Schleiermacher-

Gesellschaft in Halle, März 2009. - ISBN: 978-3-11-022053-7

Berlin/Boston: De Gruyter, 2012

S. $385-401$ 


\title{
Christentum und Judentum
}

\author{
Akten des Internationalen Kongresses der \\ Schleiermacher-Gesellschaft in Halle, März 2009
}

Herausgegeben von

Roderich Barth, Ulrich Barth und

Claus-Dieter Osthövener

De Gruyter 


\title{
Christentum und Judentum in Schleiermachers Vorlesungen über die Kirchengeschichte
}

\author{
Simon Gerber
}

"Das Christenthum steht zwar in einem besonderen Zusammenhange mit dem Judenthum; was aber sein geschichtliches Dasein und seine Abzwekkung betrifft, so verhält es sich zum Judenthum und Heidenthum gleich."

So lautet in Schleiermachers Glaubenslehre ein Leitsatz unter den Lehnsätzen aus der Apologetik, die als Vorüberlegung zur Dogmatik den Ort bestimmen sollen, den das Christentum in der Geschichte der Religionen und Religionsgemeinschaften hat. ${ }^{1}$ Innerhalb der Religionsgeschichte stellt das Christentum nicht einfach eine Fortsetzung der altrestamentlich-jüdischen Religion dar; auch wenn Christus im jüdischen Monotheismus verwurzelt ist, ist das, was mit ihm beginnt, dem Judentum gegenüber genauso etwas Neues wie gegenüber dem heidnischen Polytheismus und dem griechischphilosophischen Gottglauben.

Nun ist aber in Schleiermachers Organismus der theologischen Wissenschaft die Dogmatik nicht die einzige, ja nicht einmal die oberste Disziplin, die Wesen und Wirklichkeit des Christentums und der christlichen Gemeinschaft zum Gegenstand hat. Eine Kirche ist für Schleiermacher eine Gemeinschaft von Menschen, die auf der Grundlage eines gemeinsamen religiösen Gefühls zusammentreten, um auf geordnete Weise aufeinander zu wirken, ${ }^{2}$ im Falle der christlichen Kirche auf Grundlage des Glaubens an den Erlöser Christus. ${ }^{3}$ Die christliche Kirche beschreiben die historisch-theologischen Disziplinen, jede auf ihre Weise und aus ihrer Perspelktive. Neben der Dogmatik als systematisch zusammenhängender Darstellung der christlichen Glaubenssätze, wie sie in einer bestimmten Kirchengemeinschaft zu einer bestimmten Zeit anerkannt sind, stehen die kirchliche Statistik, die den gesellschaftlichen Zustand des gegenwärtigen Christentums

1 Friedrich Schleiermacher, Der christiiche Glaube, 2 Bde., Berlin ${ }^{2} 1830 / 31$. \$12, Leitsatz, in: Ders., Kritische Gesamtausgabe, hg.v. Hans-Joachim Birkner/Hermann Frscrerer u.a. (im Folgenden KGA abgekürzt), Bd. I/13, 1, hg.v. Rolf Schärer, Berlin/New York 2003, 102.

2 Vgl. Schleiermacher, Einleitung in die Kirchengeschichte 1806, 4. Stunde, in; KGA II/6, hg.v. Simon Gerber, Berlin/New York 2006, 12f.; DERS., Ethik 1812/13, Güterlehre, Vollkommene ethische Formen, $\$ 196-209$, in: Ders., Werke. Auswahl, hg.v. Otto Braun/Johannes BauER, Bd. 2, hg.v. Otto Braun (PhB 137), Leipzig 1913, 359-361; Ders., Der christliche Glaube ${ }^{2} \$ 6,2-4$, KGA I/ $13,1,54-58$.

3 SCHLEIERMACHER, Der christliche Glaube ${ }^{2} \$ 11$, KGA I/13,1, 93-102. 
in den Staaten der Erde beschreibt, und die Auslegung des Neuen Testaments, derjenigen Urkunde, die für alles Spätere die unhintergehbare Norm ist, weil das christliche Prinzip hier, an seinem Ursprung, seinen reinen und unvermischten Ausdruck gefunden hat. ${ }^{4}$ Und schließlich gibt es das alles verbindende Mittelstück, die whistorische Theologie im engeren Sinn«, die Kirchengeschichte. Sie betrachtet die Ausbreitung, Entwicklung und Ausdifferenzierung der christlichen Gemeinschaft und Gemeinschaften durch die Zeiten ${ }^{5}$ und zeigt so das Christentum als eine lebendige, wirkungsvolle Kraft in der Geschichte der Menschheit.

Die Grundlagen der Kirchengeschichte hat Schleiermacher in der theologischen Enzyklopädie gelegt, außerdem in einer einstündigen Vorlesung seiner Hallenser Zeit. Hier geht es um den Ort, den die Kirchengeschichte als Wissenschaft innerhalb der Theologie und der Geschichtskunde einnimmt, um die inhaltliche Teilung ihres Gegenstandes und um Fragen der wissenschaftlichen Praxis. ${ }^{6}$ In den 1820er Jahren hat Schleierma-

4 Schleiermacher, Kurze Darstellung des theologischen Studiums, Berlin ${ }^{2} 1830, \$ 81,84,95-98$, 195, in: KGA I/6, hg.v. Dirk Schmid, Berlin/New York 1998, 357f, 362-364, 393f. - Unter der dogmatischen Theologie ist hier die Sittenlehre (theologische Ethilk) inbegriffen, vgl. aaO. $\$ 223$ 231, KGA I/6, 404-407; ferner zur Vereinigung und Trennung beider dogmatischen Disziplinen DERs., Christliche Sitte 1809/10, $\$ 1-27$, in: Ders., Sämmtliche Werke (im Folgenden SW abgekïizz), Bd. I/12, hg.v. Ludwig Jonas, Berlin 1843, Beilage, 3-9; DERs., Christliche Sitte 1822/23, in: SW I/12, 2-24; DERs, Der christliche Glaube ${ }^{2}, \$ 26$, KGA I/13,1, 173-175. Vgl. dazu auch Hans-Joachim Birisner, Schleiermachers christliche Sittenlehre (TBT 8), Berlin-West 1964, 66-72.

5 Schleiermacher, Kurze Darstellung 2 , $\$ 82, \mathrm{KGA} 1 / 6,357$. Als »historische Theologie im engeren Sinn« bezeichnet Schleiermacher die Kirchengeschichte in der Überschrift vor $\$ 149$, KGA I/6, 380.

6 Die Vorlesung von 1806 gehört neben Schleiermachers Brouillon zur Ethik 1805/06, in: SchieIerMaCHer, Werke. Auswahl, Bd. 2, (s. o. Anm. 2), 73-239, zu den frühesten Dokumenten des Schleiermacherschen Systems. In ihr leitet Schleiermacher die Kirchengeschichte als wissenschaftliche Disziplin direkt aus der philosophisch-ethischen Güterlehre ab: Die Kirchengeschichte beschreibt das Symbolisieren unter dem Charakter der Individualität, tut das aber nicht wie die Ethik systematisch, sondern historisch-empirisch; sie ist also soviel wie historische Religionssoziologie. Religionssoziologie und Christentumsgeschichte fallen aber deshalb zusammen, weil erst im Christentum die Religionsgemeinschaft oder Kirche als eigene, vom Staat unabhängige ethische Form hervorgetreten sei. Vgl. Schlemermacher, Einleitung in die Kirchengeschichte 1806, 3.-4. Stunde, KGA II/6, 11-13; zum Verhältnis zwischen Ethik und Geschichte vgl. Schleikrmacher, Ethik. Letzte Bearbeitung der Einleitung (vermutlich 1816/17), \$108, in: Ders., Werke. Auswahl, Bd. 2, 549; Ders., Kurze Darstellung 2 , $\$ 35, \mathrm{KGA} \mathrm{I/6,339.} \mathrm{Demgegenüber} \mathrm{leitet} \mathrm{die} \mathrm{theologische} \mathrm{Enzyklopädie} \mathrm{die} \mathrm{Kirchen-}$ geschichte als wissenschaftliche Disziplin erst aus dem kirchenleitenden Interesse der Theologie als positiver Wissenschaft und der Notwendigkeit kirchengeschichtlicher Kenntnisse für die Amtsführung ab, vgl. aaO. $\$ 1-5,26,70,79 f$., 82, KGA I/6, 325-328, 335f., 353f., 356f.; sie stellt die Kirchengeschichte also vom latent christlichen Standpunkt der philosophischen Ethik auf einen explizit christlichen Standpunkt. - Vgl. über den Ort, clen Schleiermacher der Kirchengeschichte in der Wissenschaft und unter den theologischen Disziplinen zuweist, auch Theodor JøRGENSEN, Schleiermacher som kirkehistoriker, in: Dansk teologisk tidskrift 31 (1968), 178-214; Simon Gerber, Geschichte und Kirchengeschichte bei Schleiermacher, in: Zeitschrift für neuere Theologiegeschich- 
cher dann in Berlin zwei Überblicksvorlesungen oder Kompendien über die Kirchenund Dogmengeschichte gelesen. Um ein solches Unternehmen durchzuführen, mußte er sich unter anderem eine umfangreiche Materialsammlung mit Exzerpten und Aphorismen anlegen, im Manuskript über 200 Seiten. Hauptquelle der Exzerpte ist die vielbändige Christliche Kirchengeschichte des aus Österreich stammenden Wittenberger Historikers Johann Matthias Schröckh (1733-1808), ${ }^{7}$ ein in seiner ungeheuren Breite geradezu formloses Werk; Schröckh verbindet einen immensen Sammelfleiß mit einem fast völligen Mangel an geistiger Durchdringung und Verarbeitung. Andere Quellen sind die kirchengeschichtlichen Arbeiten des berühmten Hallenser Neologen Johann Salomo Semler (1725-1791), meist nur knappe, fast unzusammenhängend nebeneinanderstehende Bemerkungen, aber mit ihrer historischen Kritik für alles Weitere wegweisend, und die Kirchengeschichte des strengen Rationalisten Heinrich Philipp Konrad Henke in Helmstedt (1752-1809). ${ }^{8}$ Besonders für die alte Kirchengeschichte hat Schleiermacher aber auch die Quellen selbst studiert: die Kirchengeschichte Eusebs von Cäsarea und die Werke Tertullians, Clemens' von Alexandrien, Origenes' und anderer. ${ }^{9}$

Warum nahm Schleiermacher als renommierter Gelehrter von über 50 Jahren all diese Mühe für eine Disziplin auf sich, in der er es doch nie mehr auch nur annähernd so weit bringen würde wie etwa sein Berliner Kollege und weiland Hallenser Schüler August Neander? Schleiermacher sagt selbst, Zweck seiner Vorlesung sei es nicht, den gelehrten Darstellungen der Kirchenhistoriker Konkurrenz zu machen; vielmehr wolle er den Studenten eine innere Geschichte des Christentums präsentieren, eine Geschichte, in der es weniger um die einzelnen facta gehe als um die in der Geschichte wirksamen Kräfte und Ideen. So stelle sein Kolleg eine Anleitung dar, anhand deren die Studenten selbst kompetent würden, Quellen und Sekundärliteratur zu studieren; und dafür eigne sich der mündliche Vortrag auch viel besser als dicke Bücher, weil man sich mündlich freimütiger ausdrücke als schriftlich. ${ }^{10}$ Die wirksame Kraft schlechthin in der Kirchengeschichte ist aber für Schleiermacher das Christentum selbst, das von Christus ausgegangene christliche Prinzip; die Kirchengeschichte beschreibt, wie dieses Prinzip sich

te $17(2010), 34-55$.

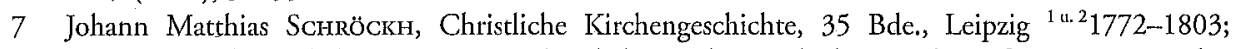
Ders./Heinrich Gottlieb Tzschirner, Christliche Kirchengeschichte seit der Reformation, 10 Bde,, Leipzig 1804-1812.

8 Johann Salomo SEMLER, Historiae ecclesiasticae selecta capita cum epitome canonum excerptis dogmaticis et tabulis chronologicis, 3 Bde., Halle 1767-1769; DERs., Versuch eines fruchtbaren Auszugs der Kirchengeschichte, 3 Bde., Halle 1773-1778; Heinrich Philipp Konrad Henke/Johann Severin VATER, Allgemeine Geschichte der christlichen Kirche nach der Zeitfolge, 9 Bde., Braunschweig ${ }^{1-4} 1800-23$.

9 Euseb von Cäsarea, Historia ecclesiastica und Vita Constantini, hg.v. Henri de Valois, Mainz 1672; DERS., Kirchen-Geschichte und Leben Constantins, übersetzt von Friedrich Andreas STroth, 2 Bde., Quedlinburg 1777; Quintus Septimius Florens Tertullian, Opera, hg.v. Johann Salomo Semler, 6 Bde., Halle 1770-1776; Clemens von Alexandrien, Opera, hg.v. John Pottrer, Venedig 1757; Origenes, Opera, hg.v. Charles Delarue OSB und Charles Vincent Delarue OSB, 4 Bde., Paris 1733-1759. Vgl, auch die Zusammenstellung in: KGA II/6, 788-794.

10 Schleiermacher, Kirchengeschichte 1821/22, Skizze der Einleitung; 1. Stunde, in: KGA II/6 (s, o. Anm. 2), 21f. 469-471; DERS., Kirchengeschichte 1825/26, in: KGA II/6, 674, 677. 
unter Gestalt der christlichen Kirche verwirklicht. ${ }^{11}$ Und hier setzt Schleiermacher sich scharf von den Kirchengeschichtlern der Aufklärung und des Rationalismus ab, denen er material ja viel verdankte: Diese folgten der sogenannte pragmatischen Methode und fragten bloß nach immanenten Ursachen und Wirkungen für die geschichtliche Entwicklung wie etwa der Gunst und Ungunst der Bischöfe am kaiserlichen Hof oder den persönlichen Ambitionen, Sym- und Antipathien der Protagonisten. Damit verlören sie aber die Mitte der Geschichte aus den Augen; am Ende komme nicht eine von einer Idee getragene, diese verwirklichende und offenbarende Geschichte heraus, sondern ein Durcheinander von Wirkungen und Gegenwirkungen. ${ }^{12}$ Abgesehen davon sei in der christlichen Kirche und ihrer Geschichte auch nur demjenigen eine dauerhafte Subsistenz und Wirksamkeit beschieden, das wirklich aus dem christlichen Prinzip und Geist hervorgegangen sei. Alles andere stelle nicht das wirksame Prinzip in der Geschichte dar, sondern das leidende Prinzip, die Materie, das »Fleisch«, also dasjenige, das der christliche Geist ergreife, innerlich durchdringe und zu seinem Organ bilde; wo es doch als eigenes Prinzip neben dem christlichen wirke, da könne es die Erscheinung der Kirche zwar trüben, aber nur vorübergehend. ${ }^{13}$

\section{2.}

Wenn Schleiermacher also das Christentum als Kirchengeschichte beschreibt, als Geschichte der Wirksamkeit des christlichen Prinzips in der Welt und als Entwicklung der Gemeinschaften unter dem christlichen Prinzip: wie stellt sich dabei das Judentum dar?

11 Schleiermacher, Kirchengeschichte $1821 / 22$, 2. Stunde, KGA II/6, 22, 474.

12 Schleiermacher, Einleitung in die Kirchengeschichte 1806, 1.-2. Stunde, KGA II/6, 9f.; DERs., Kirchengeschichte 1821/22, Skizze der Einleitung; 1.-2. Stunde, KGA II/6, 21f., 471 f.; DERs., Kirchengeschichte 1825/26, KGA II/6, 126, 669f., 674; DERs., Theologische Enzyklopädie 1831/32, Nachschrift David Friedrich Strauß, hg.v. Walter SACHs (SchlA 4), Berlin-West/New York 1987, 69; 167f. (\$65.179) - Der Begriff der pragmatischen Geschichte geht auf PoLYBIos zurück (Historiae $\mathrm{I}, 2,8 ; 35,9$ u.ö.). Im 18. Jahrhundert verstand man darunter eine Geschichte, die die Forschung nach Ursachen, Motiven und Wirkungen (also nach der kausalen Verknüpfung der Ereignisse unter Verzicht auf übernatïrliche Erklärungen) verband mit der Frage, welche praktisch-moralische Lehren die Gegenwart aus der Geschichte ziehen könne. Schleiermachers Auseinandersetzung mit der Pragmatik bezieht sich auf das Erste. Im späteren 18. Jahrhundert meinte man unter Kants Einfluß mit Pragmatils auch eine teleologische Sicht der Geschichte, die nach dem inneren Gehalt, dem notwendigen Zusammenhang und dem Ziel des Geschichtlichen fragt; diese Auffasung nähert sich derjenigen Schleiermachers. Vgl. Wilhelm BauER, Einführung in das Studium der Geschichte, Tübingen ${ }^{2} 1928,151 f$; Manfred HaHN, Art. "Geschichte, pragmatische«, in: HWP 3, Basel 1974, 401f; Gudrun Kühne-Bertram, Art. „Pragmatisch«, in: HWP 7, Basel 1989, 1241-1244, hier 1243. Zur pragmatischen Kirchengeschichtschreibung vgl. Sснröcкr (s.o. Anm. 7), Bd. 1, Leipzig ${ }^{2} 1772$, 186-188, 268-293; Karl VöLIER, Die Kirchengeschichtsschreibung der Aufklärung, Tübingen 1921, 23-36; Walter NigG: Die Kirchengeschichtsschreibung, München 1934, 138-141.

13 Schletermacher, Einleitung in die Kirchengeschichte 1806, 6.-7. und 11. Stunde, KGA II/6, 1416, 18; DERs., Christliche Sitte 1822/23, in: SW I/12, 43-45, 126, 293f; Ders., Kurze Darstellung ${ }^{2}$, $\$ 34,54,160,167,173,179, \mathrm{KGA} / 6,339,346,383,385-387,389$; DERs., Der christliche Glaube $^{2}, \$ 152,155, \mathrm{KGA}$ I/13,2, 440-443, 448-450; DERs., Theologische Enzyklopädie 1831/32, (s.o. Anm. 12), 37, 153f, 158, 167f. (\$34, 160, 167, 179). 
Zunächst: Das Judentum ist in Schleiermachers Kirchengeschichte von der zweiten Periode an, also etwa seit 300, kein sehr großes Thema. Wo es aber vorkommt, da geht es oft um christliche Feindschaft und Intoleranz gegen das Judentum. Aus der zweiten Periode der Kirchengeschichte, dem Zeitraum zwischen Konstantin und Karl dem Großen, notiert Schleiermacher, daß Ehen zwischen Christen und Juden staatlich verboten wurden, daß Juden aber das Halten christlicher Sklaven erlaubt war, daß Johannes Chrysostomus antijüdische Predigten hielt und daß Ambrosius Kaiser Theodosius den Großen davon abhielt, die Zerstörung einer Synagoge in Callinicum durch den christlichen Mob zu ahnden. ${ }^{14}$ In den germanischen Reichen in Frankreich und Spanien wurden die Juden bald verfolgt oder zwangsbekehrt, bald toleriert. Isidor von Sevilla mißbilligt die Zwangschristianisierung im Westgotenreich, befürwortet es aber, Juden ihre Kinder zu nehmen, um sie im Christentum zu erzichen, "was doch nur ein Erlaß [das heißt eine Linderung] der Gewalt war, nicht Aufhebung ihres Prinzips«. Papst Gregor der Große ist gegen die Juden in Italien duldsam; er versucht, jüdische Pächter des kirchlichen Grundbesitzes durch den versprochenen Erlaß des Pachtzinses zur Taufe zu bewegen, und hofft, daß selbst bei denen, die dieses Angebot nicht aus Glaubensüberzeugung annehmen, doch die nächste Generation wirklich christlich wird. Den Ikonenkult der Christen verspotten die Juden (Schleiermacher findet offenbar: nicht ganz zu unrecht) als Abgötterei. ${ }^{15}$ Die dritte Periode der Kirchengeschichte, die von etwa 800 bis etwa 1500 reicht, sieht die Juden im arabischen Spanien und im fränkischen Reich zunächst in hohem Ansehen. Die Kreuzfahrer unter Walter Habenichts und Peter dem Einsiedler - Schleiermacher charakterisiert sie knapp als »Gesindel — - ermorden auf ihrem Weg viele Juden. Der wissenschaftliche Aufschwung seit 1100 ergreift auch die Juden; unter ihnen entsteht, als Parallelphänomen zur lateinisch-christlichen Frühscholastik, die rabbinische Gelehrsamkeit mit Männern wie Raschi, Kimchi und Maimonides. Im 14. Jahrhundert kommt es zu heftigen Judenverfolgungen. Um 1500 schreitet die Inquisition in Spanien gegen Moslems und Juden vor, ebenso wie in Frankreich und Italien gegen Ketzer und in Deutschland und Holland gegen Hexen. ${ }^{16}$

Wenn Schléiermacher über Glaubenszwang und Schikanen von Christen gegen Juden berichtet, und zwar im Ton scharfer Mißbilligung, ${ }^{17}$ so steht er mit der aufgeklärtpragmatischen Kirchengeschichtschreibung, aus der seine Kenntnisse kommen, in voller

14 SCHLEIERMACHER, Kollektaneen 300; 306, in: KGA II/6 (s. o. Anm. 2), $207 \mathrm{f}$.

15 SChleiermacher, Kirchengeschichte 1821/22, 59. Stunde, KGA II/6, 534; DERs., Kirchengeschichte 1825/26, KGA II/6, 139, und DERs., SW I/11, hg.v. Eduard BonNELl, Berlin 1840, 367f., 371; DERs., Kollektaneen 471; 537; 550; 570; 588; 1143, KGA II/6, 244, 262. 265. 268. 277. 428. Vgl. zu Gregors Projekt auch Schleiermachers Bemerkung: "Aber die Römische Kirche glaubt schon immer etwas zu haben, wenn sie nur etwas Äußeres hat von dem Grundsatz aus: daß man nicht wissen kann, was dadurch mit der Zeit wird " (Schlemermacher, Kirchliche Statistik 1827, 15. Stunde, in: KGA II/16, hg.v. Simon Gerber, Berlin/New York 2005, 243).

16 SCHLeIermacher, Kirchengeschichte 1821/22, 85. und 91. Stunde, KGA II/6, 608. 625; DERs., Kirchengeschichte 1825/26, SW I/11, 481. 545; DERs., Kollektaneen 626; 710; 754; 810, KGA III/6, 288. 324. 337. 351.

17 Vgl. auch Schlemermacher, Kirchliche Statistils 1827, 20. Stunde, KGA II/16, 259f.; Ders., Katholische Kirche 45, KGA II/16, 72 f. 
Übereinstimmung auch der Beurteilung. Semler schreibt in seinen Zenturien vom fünften Jahrhundert an fast jedes Mal von Verleumdungen, Gewalttaten und bürgerlichen Zwangsmaßnahmen gegen die Juden, auch von Übertritten zum Christentum aus Opportunismus ${ }^{18}$ gelegentlich schließlich auch von richterlicher Fairneß und Schutzmaßnahmen des christlichen Klerus zugunsten der Juden. ${ }^{19}$ Noch reicheres Material darüber findet sich bei Schröckh. ${ }^{20}$ Um ein waches, selbstkritisches Auge für die mancherlei judenfeindlichen Garstigkeiten in der Geschichte der christlichen Kirche zu haben, bedurfte es keiner "Erneuerung des Verhältnisses von Christen und Juden ${ }^{21}$ und keiner »Theologie nach Auschwitz «. 22

Über eine geschichtstheoretische Einordnung des Phänomens Judenfeindschaft sagt Schleiermachers Kirchengeschichte nichts Spezielles. Nach seinem Grundsatz, daß die Mißbildungen und Rückschritte in der Entwicklung der Kirche immer aus dem Menschen, insofern er vom christlichen Geist noch nicht voll durchdrungen ist, kommen, bei neubekehrten Individuen und Völkern insbesondere aus ihrem unchristlichen Vor-

18 Semler, Versuch (s.o. Anm. 8), Bd. 1, Halle 1773, 93f. 154-156. 211f. 245f. 286. 376. 470-482. 587. 591-593. 651-653; Ders., Versuch, Bd. 2, Halle 1774, 19.61-63.

19 Semler, Versuch, Bd. 1, 155f. 286. 482f. 591f.; Ders., Versuch, Bd. 2, 61.

20 Vgl. das Register: Schröcкн (s.o. Anm. 7), Bd. 35, Leipzig 1803, 202f. Schröckh ist im Vergleich mit Semler noch mehr Polyhistor und weniger kritischer Historiker; doch wo er Berichte z.B. über jüdische Untaten an Hostien und Christenkindern zitiert und auf ihren Wahrheitsgehalt prüft, kommt auch er zu einem negativen Ergebnis. - Vgl. auch HENke/VATER: Allgemeine Geschichte (s.o. Anm. 8), Bd. 9, Braunschweig 1823, 567, bei dem das Thema eine geringere Rolle spielt.

21 So der programmatische Beschluß der rheinischen Landessynode vom 11.1.1980 (Handreichung für Mitglieder der Landessynode, der Kreissynoden und der Presbyterien in der Evangelischen Kirche im Rheinland 39, Mülheim [1980], 9-11, auch abgedruckt in: Günther Bernd GINZEL (Hg.), Auschwitz als Herausforderung für Juden und Christen [Tacheles: Zur Sache 1], Heidelberg 1980, 402-407), in dem es u.a. heißt, die Kirche sei »durch Jesus Christus in den Bund Gottes mit seinem Volk hineingenommen«; gemeint ist offenbar der Sinaibund. Knapp und treffend dazu noch immer die Erwägungen der Bonner und Münsteraner Theologieprofessoren (epd-Dokumentation 1980, Nr. $42,14-17)$.

22 Vgl. z. B. Bertold Klappert, Die Juden in einer christlichen Theologie nach Auschwitz, in: Ginzer (s.o. Anm. 21), 481-512, wo es u.a. heißt: "Christliche Theologie nach Auschwitz ist darin auf das Judentum als den Zeugen der messianisch-apokalyptischen Hoffnung angewiesen, daß sie sich lösen muß von einem sterilen Christozentrismus oder Christomonismus [...]. Nur der gekreuzigte und der in Auschwitz mitleidende und vernichtete Gott kann helfen. Es liegt an der Sache selbst, daß wir hier nicht mehr von mitgebrachten Vorstellungen über Gott, sondern nur noch von den jüdischen Zeugen Gottes in Auschwitz herkommen können« (495. 498). Das ist sicher mit brennendem Herzen gesprochen, steht aber in genauem Gegensatz zur ersten Barmer These: "Wir verwerfen die falsche Lehre, als könne und müsse die Kirche als Quelle ihrer Verkündigung außer und neben diesem einen Worte Gottes [scil. Jesus Christus] auch noch andere Ereignisse und Mächte, Gestalten und Wahrheiten als Gottes Offenbarung anerkennen" (Kurt Dietrich SchmidT, Die Bekenntnisse und grundsätzlichen Äußerungen zur Kirchenfrage, Bd. 2. Das Jahr 1934, Göttingen 1935, 93). Tatsächlich ist "Christomonismus« eben das, was Paul Althaus der Barmer Erklärung vorgeworfen hat, vgl, Paul Althaus, Dic christliche Wahrheit, Bd. 1, Gütersloh ${ }^{2} 1949,68-73$; Ernst WoLp, Barmen (BEvTh 27), München ${ }^{2} 1970,94-103$. Freilich wollte Althaus geltend machen, daß Christus der Offenbarer des Evangeliums, aber nicht des Gesetzes und der Ordnungen sei, während es Klappert eher um eine Ergänzung der. Heilsoffenbarung Christi in der Geschichte geht. 
leben, ${ }^{23}$ müßten Gewalt und Zwang gegen Andersgläubige Erscheinungsformen der fleischlichen Laster sein, die mit der intensiven Steigerung des Christentums in den Menschen aber allmählich aufhören. Als besondere Ursache sieht Schleiermacher vielleicht eine Verwirrung der ethischen Formen ${ }^{24}$ (also etwa das, was die Confessio Augustana das »Ineinanderwerfen und Mengen der zwei Regimenter« nennt), ${ }^{25}$ das heißt den Versuch, religiöse Fragen und Differenzen mit Mitteln und Sanktionen des Staates zu bereinigen.

\section{3.}

Kehren wir nach diesem Streifzug durch die zweite und dritte Periode der Kirchengeschichte wieder an den Anfang zurück, zu Schleiermachers Vorhaben, eine von einer Idee getragene Geschichte zu erzählen! Macht so eine Idee den Historiker nicht befangen? Ziemlich zu Beginn des ersten Kompemdiums von 1821/22 legt Schleiermacher dar, daß jede Geschichte mindestens latent immer schon von der Anschauung des Betrachters bestimmt sei. Eine neutrale, wirklich unvoreingenommene Geschichte gebe es gar nicht; auch sei es unmöglich, die Geschichte zunächst unparteiisch und unbefangen zu betrachten und sich erst dann anhand der objektiven Tatsachen seinen Standpunkt zu wählen. Diese Abhängigkeit der Geschichte von der subjektiven Sicht des Anschauenden, seinen Sympathien und Antipathien angesichts des Gegenstandes, sei bei der Kirchengeschichte noch stärker als bei einer politischen Geschichte gegeben. Man müsse also bei einer Darstellung der Kirchengeschichte immer zuerst den Standpunkt des Darstellenden kennenlernen, und im Gegensatz zu vielen anderen wolle er, Schleiermacher, damit auch gar nicht hinter dem Berg halten. Sein Glaubensbekenntnis sei: 1) Christus sei nicht ein Fortsetzer außerchristlicher Anfänge im Judentum oder im Heidentum, auch kein Reformator des Judentums; das Christentum fange erst mit dem an, was in Christus als Ursprüngliches gesetzt gewesen sei. Daraus folge 2), daß dieses Ursprüngliche in Christus etwas Göttliches, eine neue Offenbarung sei, dazu bestimmt, zum Eigentum der ganzen Menschheit zu werden. ${ }^{26}$

Wir sind also inhaltlich wieder bei dem anfangs zitierten Leitsatz aus der Glaubenslehre von der Selbständigkeit der christlichen Religion auch gegenüber dem Judentum angelangt; nur gilt das hier interessanterweise nicht als wissenschaftlicher Satz aus der religionsphilosophischen Apologetik, ${ }^{27}$ sondern als Glaubenssatz. Die Vorlesung wurde in zeitlicher Nähe zur ersten Auflage der Glaubenslehre und zur Dialektik von 1822 gehal-

23 Vgl. Schleiermacher, Einleitung in die Kirchengeschichte 1806, 5. Stunde, KGA II/6, 13; DERs., Kirchengeschichte 1821/22, 3. Stunde, KGA II/6, 22f. 474-476; DERs., Kirchengeschichte 1825/26, KGA II/6, 682; DERs., Christliche Sitte 1826/27, SW I/12, 209; DERS., Der christliche Glaube ${ }^{2}$, $\$ 148, \mathrm{KGA} \mathrm{I} / 13,2,427-430$; DERs., Theologische Enzyklopädie 1831/32, \$83, (s.o. Anm. 12), 86f.

24 Vgl. dazu Schleirrmacher, Einleitung in die Kirchengeschichte 1806, 6.-7. Stunde, KGA II/6, $14-16$.

25 Confessio Augustana XXVIII, 12, in: BSLK, Göttingen ${ }^{8} 1979,122$.

26 Schlemermacher, Kirchengeschichte 1821/22, 2. Stunde, KGA II/6, 22. 471-474.

27 Vgl. Schleigrmacher, Kurze Darstellung $\$ 21$. 24. 39. 43-46, KGA I/6, 334f. 340f. 342 f. 
ten, die die Eigenständigkeit des christlichen Glaubens und der Religion überhaupt gegenüber dem philosophischen System und der Wissenschaft betonen. ${ }^{28}$ Vielleicht geht es Schleiermacher hier also darum, die Kirchengeschichte als theologische Disziplin im kirchenleitenden Interesse auf ein allein kirchlich-religiöses Fundament zu stellen. - Das zweite Kompendium wiederholt das »Glaubensbekenntnis« inhaltlich; Schleiermacher sagt, daß er "weniger als andere Theologen das Christenthum für eine Fortsezung des Judenthums" halte. ${ }^{29}$

In jüngerer Zeit haben Klaus Beckmann und noch mehr Matthias Wolfes Schleiermacher für seine scharfe Grenzziehung zwischen Altem Testament und Judentum einerseits, Christus und dem Christentum andererseits kritisiert, die mit einer Abwertung von ersterem einhergehe. ${ }^{30}$ Für Wolfes ist Schleiermachers ungünstige Beurteilung der alttestamentlich-jüdischen Religion gar mitverantwortlich für das Versagen der evangelischen Kirchen vor dem mörderischen Judenhaß des 20. Jahrhunderts. ${ }^{31}$ Auch nehme Schleiermachers Christusbild gänzlich unhistorische Züge an. ${ }^{32}$ Beckmann konstatiert, daß Schleiermacher zwar kein Anhänger der sogenannte Substitutionstheorie sei, wonach Israel (beziehungsweise das Judentum) als Gottesvolk durch die Kirche abgelöst worden sei, daß er aber die besonders in seiner reformierten Konfession gepflegte Vorstellung von einem das biblische Israel und dic Kirche umfassenden Gottesvolk nicht teile. ${ }^{33}$ Allerdings basiert die von Beckmann als antijudaistisch gescholtene Substitu-

28 Schleiermacher, Der christliche Glaube nach den Grundsäzen der evangelischen Kirche im Zusammenhange dargestellt, 2 Bde., Berlin 1821/22, $\$ 1,4 ; 2,2 ; 31,1 ; 38$, in: KGA I/7, 1, hg.v. Hermann Perter, Berlin-West/New York 1980, 12. 15f. 109-112. 127f.; Ders., Dialektik 1822, 50.-51. Stunde, in: KGA II/10,1, hg.v. Andreas ARNDT, Berlin/New York 2002, 265-267, und Bd. II/10,2, Berlin/New York 2002, 565-571; vgl. auch DeRs., Christliche Sitte 1822/23, SW I/12, 30f. 75. $87-93$.

29 Schleiermacher, Kirchengeschichte 1825/26, KGA II/6, 673. 679. Vgl. dazu auch die Beiträge von Jan Rohls und Notger Slenczka in diesem Band.

30 Klaus Beckmann, Die fremde Wurzel (FKDG 85), Göttingen 2002, 34-103. 312-323; Matthias Wolfes, Schleiermacher und das Judentum. Aspekte der antijudaistischen Motivgeschichte im deutschen Kulturprotestantismus, in: Aschkenas 14 (2004), 485-510; DERs., Öffentlichkeit und Bürgergesellschaft, Bd. 2 (AKG 85,2), Berlin/New York 2004, 360-390; DERs., Schleiermacher and Judaism. On the Relationship Between Protestant Theology and Emancipation Politics in the Late Eighteenth and Early Nineteenth Century, in: Hans Dierkes/Terrence N. Tice/Wolfgang Virmond $(\mathrm{Hg}$ ), Schleiermacher, Romanticism, and the Critical Arts. A Festschrift in Honor of Hermann Patsch (New Athenaeum/Neues Athenaeum. 8), Lewiston/Queenston/Lampeter 2007/08, 305-326.

31 Wolfes, Schleiermacher und das Judentum, 488f.; Ders., Öffentlichkeit, Bd. 2, 326-329; Ders., Schleiermacher and Judaism, 307; ähnlich auch schon Hans-Joachim IwAND: Die Kirche und die Juden, in: JK 12 (1951), 105f. - Das einzig Wahre dazu hatte Schleiermacher schon 1799 wenigstens grundsätzlich gesagt, daß nämlich die Frage der Toletanz in Staat und Gesellschaft und die nach der religiös-dogmatischen Wahrheit grundsätzlich nicht miteinander vermengt werden dürften; vgl. [Schleiermacher,] Briefe bei Gelegenheit der politisch theologischen Aufgabe und des Sendschreibens jüdischer Hausväter, Berlin 1799, 11-19, in: KGA I/2, hg.v. Günter MвCKENsTock, Berlin-West/New York 1984, 331 338. Vgl, dazu auch den Beitrag von Hans-Martin Kirn in diesem Band.

32 Wolres, Schleiermacher und das Judentum, 502; DERs., Schleiermacher and Judaism, 319.

33 BeckManN, aaO., 99. 102f. 
tionstheorie $^{34}$ ja gerade auf der Vorstellung einer unmittelbaren Kontinuität zwischen dem biblischen Israel und der christlichen Kirche und auf der direkten Parallelisierung zwischen Sinaibund und Evangelium. ${ }^{35}$ Für Schleiermacher ist das Judentum nicht bloß eine Vorform oder mißratene Nebenform des Christentums; er hat es als eine auch gegenüber dem Christentum eigenständige Gestalt der monotheistischen Religion ernstgenommen, $\mathrm{Ob}$ seine Charakterisierung des Judentums diesem tatsächlich gerecht wird, ${ }^{36}$ ist freilich eine andere Frage. Das gilt aber zum Beispiel auch für seine Beurteilung der Ostkirchen. ${ }^{37}$

Worum geht es Schleiermacher also bei seinem Glaubensbekenntnis, das er der Kirchengeschichte zugrundelegt? Es ist die Alleinmittlerschaft Christi, seine Einzigartigkeit als Stifter des neuen menschlichen Gesamtlebens. Wenn von einer göttlichen »Offenbarung in Christus die Rede ist, meint das freilich nichts Übernatürliches, von Gott unmittelbar und außer dem Kausalzusammenhang der Natur Gewirktes, unfehlbar Wahres. Offenbarung ist nach der Glaubenslehre vielmehr so etwas wie religiöse Spontaneität oder Kreativität, also etwas, was so nicht aus der gegenseitigen Einwirkung der Menschen aufeinander abgeleitet werden kann; in diesem Sinne kann auch der Ursprung anderer Religionen Offenbarung genannt werden. ${ }^{38}$ Schleiermachers Glaubensbekennt-

34 Eine Art Substitutionstheorie gegeniiber Israel hatte auch schon das frühe Judentum, vgl. Hos 1,7 (als Glosse zu 1,6); Ps 78,67f:; 2Chr 13,4-11.

35 Vgl, zu dem Problem auch Werner Wiesner, Art. "Bund V. Alter und neuer Bund, dogmatisch", in: $\mathrm{RGG}^{3}$ 1, Tübingen 1957, 1521-1523. Bei Paulus entspricht der positiven Willenskundgebung Gottes an Israel in der Tora auf Seiten der anderen Völker eben nicht das Evangelium, sondern die Selbstoffenbarung Gottes durch die Schöpfung; das Evangelium ist gegenüber beiden das Neue (Röm 1, 14-3,30). Daß im Übrigen aus dem positiven Anknüpfen an die alttestamentlichisraelitische und jüdische Tradition nicht unbedingt auch eine günstige Beurteilung des Judentums folgt, zeigt schon das Matthäusevangelium.

36 Vgl, besonders die vieldiskutierte Passage: [Schlemermacher,] Über die Religion, Berlin 1799, 286 291, in: KGA I/2, 314-316; dazu auch Arnulf von Schelihas Beitrag in diesem Band. Daß hier indessen der Gott des Judentums als maschineller Schicksalsverwalter erscheine und der Freiheitsbegriff aufgelöst werde (so WoLFEs, Schleiermacher und das Judentum, 505; DERs., Schleiermacher and Judaism, 321), stimmt nicht; Schleiermacher bestimmt das Verhältnis zwischen Mensch und Gott nach jüdischem Verständnis ja gerade als beständigen Dialog von freier Tat des Endlichen und »Reaction des Unendlichen gegen Jedes einzelne Endliche«. Das Wesen des Judentums ist fïr Schleiermacher danach also der Glaube an den Tun-Ergehens-Zusammenhang, wie er sich im Alten Testament besonders in den deuteronomistischen Passagen der Bücher Den bis 2 Kön und Jer und in 1-2Chr äußert, im neuen Testament etwa in der Frage der Jünger über den Blindgeborenen (Joh 9,2).

37 Vgl. Schlemermacher, Kirchengeschichte 1821/22, 59. Stunde, KGA II/6, 536; Ders., Kirchliche Statistik 1827, 5. und 16. Stunde, KGA II/16, 204f. 244; DERs., Kirchliche Statistik 1833/34, 5. und 9.-10. Stunde, KGA II/16, 476-478. 487f.; dazu auch Simon Gerber, Schleiermacher und die Kirchenkunde des 19. Jahrhunderts, in: Zeitschrift für neuere Theologiegeschichte 11 (2004), 183-214, hier 187f; DERs., Kirchliche Statistik als Soziologie des Christentums, in: Andreas Arndt/Ulrich BARTH/Wilhelm GRÄB (Hg.), Christentum - Staat - Kultur. Alsten des Kongresses der Internationalen Schleiermacher-Gesellschaft in Berlin, März 2006 (SchlA 22), Berlin/New York 2008, 443-457, hier 449-451.

38 Schletermacher, Der christliche Glaube ${ }^{1}, \$ 19,2$ f, KGA I/7, 1, 72-77. Vgl, auch [Ders., Über die Religion, 287, KGA 1/2, 314: wich haße in der Religion diese Art von historischen Beziehungen, ihre Nothwendigkeit ist eine weit höhere und ewige, und jedes Anfangen in ihr ist ursprünglich«. In 
nis meint also keinen exklusiven, übernatürlichen Offenbarungscharakter der christlichen Religion, sondern zweierlei: die religiöse Originalität und die Universalität Christi und damit auch der durch ihn gestifteten, auf ihm gründenden Glaubensgemeinschaft. Christus ist kein Lehrer natïrlicher Tugend und Weisheit, auch kein Verbesserer einer schon vorhandenen Religion. Mit ihm beginnt vielmehr etwas Neues, und dieses Neue, das nur in ihm und durch seine Mittlerschaft da ist, bezieht sich auf alles Bisherige in gleicher Weise; darum ist es keine Substitutierung des Judentums, jedenfalls nicht mehr als irgend einer anderen Religion.

Schleiermacher ist auch der Meinung, daß eine Kirchengeschichte, die nicht auf einem solchen christlich-religiösen Fundament steht, eine Absurdität wäre: Für einen Verächter der Religion ist das Christentum gar kein positives Element der Weltgeschichte, sondern ein ethischer Irrtum, der verschwinden muß; ein rein negative Idee kann aber nicht Gegenstand der Geschichte, der positiven Weltentwicklung sein. Nimmt man das Christentum wiederum nicht wie er, Schleiermacher, als etwas Neues, sondern als Fortsetzung etwas schon Vorhandenen wie des Judentums, dann ist seine Geschichte eben kein eigener organischer Abschnitt der Weltentgeschichte, sondern nur ein mehr oder weniger willkürlich vorgenommener Ausschnitt aus einer übergeordneten Geschichte. ${ }^{39}$

Kirchengeschichte (und historische Theologie insgesamt) zu betreiben ist bei Schleiermacher also nichts anderes als den Glaubenssatz von der religiösen Originalität und Alleinmittlerschaft Christi auf der Grundlage der philosophisch-ethischen Kulturtheorie historisch zu explizieren.

Wie sieht nun Schleiermachers historische Konstruktion der Neuheit und Originalität Christi und seiner Gemeinschaft gegenüber allem Bisherigen aus? Zunächst: Schleiermacher leugnet nicht die nationale und kulturelle Verwurzelung Christi und des frühen Christentums im Judentum; auch, sagt er, hätte Christus nicht ebensogut in einem polytheistischen wie im jüdisch-monotheistischen Kontext auftreten können. ${ }^{40}$ Das zweite Kompendium wirft sogar einen Blick auf das Judentum dieser Zeit: Von den jüdischen Gruppen seien die elitären Sadduzäer (deren Arroganz sich schon darin äußere, daß

diesem Sinne hat nicht nur Schleiermachers Christusbild unhistorische Züge (s. o. Anm. 32), sondern sein Bild jedes Religionsstifters und jedes Anfangs in der Reliigonsgeschichte.

39 Schleirrmacher, Einleitung in die Kirchengeschichte 1806, 4. Stunde, KGA II/6, 12; Ders., Ethik 1812/13, Einleitung, $\$ 47$, in: DERs., Werke. Auswahl, Bd. 2 (s.o. Anm. 2), 250; DERs., Kirchengeschichte 1821/22, 2. Stunde, KGA II/6, 22, 471f.; DERs., Kirchengeschichte 1825/26, KGA II/6, 125f. 670f. 678f.; DERs., Kurze Darstellung 2 , \$78-80, KGA 1/6, $356 \mathrm{f}$.

40 Schlemermacher, Kirchengeschichte 1825/26, KGA II/6, 673; DERs., Christliche Sitte 1826/27, in: SW I/12 (s. o. Anm. 4), 292f.; Ders., Der christliche Glaube ${ }^{2}, \$ 12$, 1f.; 132,3, KGA I/13,1, 103105, und Bd. I/132, 340f; DERs., Theologische Enzylklopädie 1831/32, \$83, (s.o. Anm. 12), 86; Ders., Einleitung ins Neue Testament 1831/32, $\$ 9$, in: SW I/8, hg.v. Gcorg Wolde, Berlin 1845, 26f; Ders., Das Leben Jesu 1832, 2. und 43. Stunde, in: SW I/6, hg.v. Karl August Rütenik, Berlin 1864, 12f. 305. Vgl. dazu auch den Hermann Fischers Beitrag in diesem Band. 
ihnen die bei den einfachen Gläubigen immer entscheidende Frage nach der äußeren Lebensführung völlig gleichgültig gewesen sei) und die ganz konventikehaften Essener klein und unbedeutend gewesen; die (schon zu Schleiermachers Zeit beliebte) These von einem besonderen Zusammenhang Christi mit den Essenern sei haltlos. Vor allem die Pharisäer seien es gewesen, unter denen Christus und die Apostel Anhänger gefunden hätten. In der jüdisch-apokalyptischen Literatur findet Schleiermacher schon eine frühe Form der kabbalistischen Vorstellung von der Himmelfahrt und Wiederkehr der Seele Adams in Henoch, Mose, Elia und dem Messias. Die Apostel hätten sich nun in ihrer Predigt nicht in erster Linie auf die Auferstehung und Himmelfahrt Christi berufen, denn hätten sie das getan, dann hätten sie unfehlbar alle kabbalistischen Juden davon überzeugt, daß Christus der erwartete Wiedergänger sei. Der Hauptanknüpfungspunkt für die Apostel seien vielmehr die im Volk lebendigen messianischen Hoffnungen gewesen; diese mußten freilich erst vom Grobsinnlichen gereinigt werden, gaben aber dem Christentum einen ersten Standpunkt in der geschichtlichen Wirklichkeit. ${ }^{41}$

Die Urgemeinde lebt und webt noch ganz im Judentum, nimmt am jüdischen Kultus teil und hat daneben ihren eigenen Kultus am Sonntag. Die jüdische Umwelt nimmt sie als eine messianische Privatsynagoge neben anderen wahr. Wer zu ihr stößt, bedarf noch nicht einmal einer besonderen Unterweisung; ihr einziges Proprium gegenüber dem sonstigen Judentum ist das Bekenntnis zu Christus. Die hohepriesterliche Obrigkeit schreitet zwar gelegentlich gegen die Urgemeinde ein, aber nur, weil diese ihr Todesurteil über Christus kompromittiert; ansonsten stehen Glaube und Praxis der Urgemeinde mit keinem jüdischen Dogma in Widerspruch. Selbst die gesetzes- und tempelkritische Rede des Stephanus (Apg 7,8-53) bewegt sich für Schleiermacher noch im Rahmen der jüdischen Hoffnung, daß der Messias die beschwerlichen Gesetzesvorschriften aufheben werde. ${ }^{42}$

Wann und aus welchem Anlaß kommt es nun zum Bruch und zur Exkommunikation der Christen aus der jüdischen Synagoge? Danach fragt Schleiermacher nicht, sondern er weist darauf hin, daß es den Christen selbst allmählich selbst zum Bewußtsein kommt, daß das Christentum seinem Wesen nach etwas dem Judentum gegenüber Neues und Eigenes ist. Anlaß dazu ist die Aufnahme zunächst von Samaritern und Proselyten, dann von Heiden in die Gemeinde und die Frage, ob von ihnen allen das Halten des jüdischen Gesetzes verlangt werden müsse oder nicht. ${ }^{43}$ Zum liberalen Gegengewicht gegen das streng jüdische Jerusalem wird Antiochia; aber auch die antiochenische Freisinnigkeit wurzelt im Judentum, nämlich im hellenistischen Judentum, das es von jeher mit dem Gesetz weniger genau nahm als das palästinische. Mit der Philippika des

41 SCHLeIERMACHER, Kirchengeschichte 1825/26, KGA II/6, 703f. Der christliche Chiliasmus ist für Schleiermacher - wie auch für Confessio Augustana XVII, 5, in: BSLK 8 , (s.o. Anm. 25), 72 - unkritisch rezeptierter jüdischer Messianismus (SchleIERmacher, Kirchengeschichte 1821/22, 14. und 23. Stunde, KGA II/6, 36. 56; DERs., Kirchengeschichte 1825/26, KGA II/6, 127. 131. 716f.; DERs., Kollektaneen 42; 126; 128; 138, KGA II/6, 151. 168f: 171).

42 Schumerrmacher, Kirchengeschichte 1821/22, 9.-10. Stunde, KGA II/6, 27f. 491-493.

43 Vgl. Schleiermacher, Christliche Sitte 1822/23, SW I/12, 181: "So lange das Christenthum allein unter den Juden bestand, konnte die Frage gar nicht entstehen, ob die Verbindlichkeit des mosaischen Gesezes fortdaure, oder nicht، ، 
Paulus gegen Petrus und Barnabas (Gal2,14-21) beginnt die notwendige Emanzipation des Christentums von dem geistig-kulturellen Zusammenhang, innerhalb dessen es seine erste Gestalt angenommen hat: Die Befolgung des Gesetzes habe auch für den Judenchristen keinen religiösen Wert mehr, sie mache »kein Verhältniß mehr [...] zwischen Gott und ihm«, sondern gehöre lediglich zu seiner nationalen Identität. ${ }^{44}$

Für Schleiermacher ist klar, daß in diesem Zwiespalt Paulus recht hat und seinem Konzept die Zukunft gehört. Dennoch versucht er, auch der anderen Seite gerecht zu werden: Beiden ging es, wie er in der christlichen Sittenlehre ausführt, darum, das ursprüngliche Christentum zu bewahren. Die Judaisten

"gingen von der Idee aus, daß das Christenthum im Judenthume entstanden, und also nur eine Modification desselben sei. [... ] Folglich bedürfe es der Wiederherstellung, nämlich der Rülkkbildung zu der Vorstellung, das mosaische Gesez sei für alle Christen ohne Unterschied verbindlich."

Paulus hingegen sah die Selbständigkeit des Christentums vom Judentum als ursprünglich an, nur sei sie dadurch in Vergessenheit geraten oder noch nicht zum Bewußtsein gekommen, daß zunächst eben alle Christen Juden gewesen seien und als solche das Gesetz gehalten hätten. ${ }^{45}$ Letzten Endes sei es aber gut gewesen, daß beide Seiten ein Abkommen gefunden hätten, das beide Auffassungen ohne wechselseitige Verdammung nebeneinander bestehen ließ (hier denkt Schleiermacher an die Abkommen Gal2, 1-10; Apg 15, 1-35). Eine einseitige Durchsetzung der liberalen Position Pauli nach Art der späteren Konzile mit ihren Mehrheitsbeschlüssen und Anathemata gegen die Unterlegenen hätte den Gemeingeist des palästinischen Judenchristentums vergewaltigt und die Kirche gespalten. So aber konnte Paulus mit seiner gesetzesfreien Heidenmission fortfahren und die Basis dafür schaffen, daß seine bessere Einsicht allmählich zum Gemeingut der ganzen Kirche werden konnte. ${ }^{46}$

Es gehört zu den Grundgegebenheiten der Kirchengeschichte, daß sich der christliche Geist, je nach der individuellen, sprachlich-kulturellen und nationalen Verschiedenheit seiner Organe, in immer neuen Gestalten verwirklicht; ${ }^{47}$ Christentum, das hatte Paulus erkannt, gibt es nicht nur im Kontext der jüdischen Kultur.

44 Schleiermacher, Kirchengeschichte 1821/22, 10.-11. Stunde, KGA II/6, 28-30. 493-496.

45 SchleIermacher, Christliche Sitte 1822/23, SW I/12, 180 f.

46 Schleiermacher, Kirchengeschichte 1821/22, 11. Stunde, KGA II/6, 30. 496f. Vgl. Ders., Der christliche Glaube ${ }^{2}, \$ 150,1$, KGA I/13,2, 435f., wonach die Urkirche nur wegen des damals noch kräftigen gemeinschaftbildenden Prinzips nicht in eine juden- und eine heidenchristliche Partialkirche zerfiel.

47 Schleiermacher, Christiche Sitte 1809/10, $\$ 148$, SW I/12, Beilage, 50f.; ders., Kirchengeschichte 1821/22, 3.-4. Stunde, KGA II/6 22f, 474-479; DERS., Christliche Sitte 1822/23, SW I/12, Beilage, 155, und Bd. I/12, 57-64. 135. 304f. 567f. 574; DERs., Christliche Sitte 1824/25, in: SW I/12, 64; DERS., Kirchengeschichte 1825/26, KGA II/6, 682-686; DERs., Christliche Sitte 1826/27, SW I/12, 419; DERS., Kirchliche Statistik 1833/34, 2. Stunde, KGA II/16, 468f. 
Die Unsicherheit der christlichen Kirche über ihren Ort in der Religionsgeschichte, aber auch in der menschlichen Kulturwelt überhaupt, dauert aber noch die ganze Periode bis zu Konstantin an. Sie äußert sich nicht nur in einer gelegentlich aufbrechenden Totalopposition gegen Staat und Gesellschaft, ${ }^{48}$ sondern auch in judaisierenden und ethnisierenden Überfremdungen des genuin Christlichen. Eine Extravaganz ins Jüdische stellt die ebionitisch-nazoräische Häresie dar, die Christus ganz in die israelitisch-jüdische Geschichte einordnet und zu einem bloßen Propheten degradiert. Diese Hairesis sei nichts anderes als die palästinische Urgemeinde, die im Jüdischen Krieg zwischen die Fronten geriet, ins Ostjordanland floh und so den Zusammenhang mit der Ökumene verlor. Von allen Entwicklungen abgeschnitten, erstarrte sie in ihrem Judentum. Als profiliertesten Vertreter dieser Richtung nennt Schleiermacher den (meist als Gnostiker geltenden) Kerinth mit seiner für das Judenchristentum charakteristischen Neigung zum Natürlichen statt Wunderbaren und zur Buchstäblichkeit statt Geistigkeit: Kerinth erklärt die fortdauernde Befolgung des jüdischen Gesetzes für notwendig und glaubt nicht an eine übernatürliche Erzeugung Jesu. Er deutet die Taufe Jesu adoptianisch, er bestreitet, daß der Logos im Fleisch gelitten habe (Christus sei durch den Logos inspiriert nach Analogie der Propheten, also könne dieser ihn wieder verlassen, und da der jüdische Opferkult damals ja noch fortdauerte, kommt dem Tod Christi für Kerinth wohl ohnehin keine Heilsbedeutung $\mathrm{zu}$ ), und er erwartet ein irdisches tausendjähriges Reich (er vertritt also eine diesseitige Eschatologie und ein buchstäbliches Verständnis der biblischen Verheißungen, wobei Christi Auferstehung mit seiner kommenden sichtbaren Wiederkehr identifiziert wird). ${ }^{49}$ Immerhin urteilte Origenes milde über dieses Judenchristentum: Es sei eine Dürftigkeit, aber keine eigentliche Irrlehre. ${ }^{50}$

Dem Ebionitismus oder Nazoräertum als Überfremdung des Christentums durch das Jüdische entspricht auf heidnisch-hellenischer Seite der Gnostizismus:

„Gnostisches Grenzvermischung gegen das Heidenthum[,] die Nazaräer gegen das Judenthum «,

schreibt Schleiermacher. ${ }^{51}$ Daß der Extravaganz ins Jüdische eine ebensolche ins Heidnische entspreche, ist aber nicht erst aus dem Phänomen des Gnostizismus abgeleitet; es

48 SChLeIERmaCHER, Kirchengeschichte 1825/26, KGA II/6, 129. 696. 715f. 723; Ders., Kollektaneum 976, KGA II/6, 391.

49 SCHLEIERMACHer, Kirchengeschichte 1821/22, 14.-15. Stunde, KGA II/6, 35-38; DERs., Kirchengeschichte 1825/26, KGA II/6, 130f. 706-708; Ders., Kollektaneen 119; 126; 128, KGA II/6, 167169. Daß Kerinth, wie TheodoRET (Haereticarum fabularum compendium II, 3, in: MPG 83, Paris 1864, 389) behauptet, die Welt - typisch gnostisch, aber ganz unjüdisch - nicht für Gottes Werk gehalten habe, hält Schleiermacher fürr unzutreffend (SCHLEIERMACFIER, Kirchengeschichte 1825/26, SW I/11, 104f; DERs., Kollektaneum 126, KGA II/6, 168).

50 SCHLEIERMACHER, Kirchengeschichte 1825/26, SW I/11, 79f,; DERs., Kollektaneum 74, KGA II/6, 157 (nach OrIgenes, Commentarii in Matthaeum XVI, 12, in: GCS 40 [Origenes X], hg.v. Ernst Benz/Erich Klostermann, Leipzig 1935, 511-513).

51 Schlemermacien, Kollektaneum 1, KGA II/6, 143; vgl. Ders., Kirchengeschichte 1821/22, 15., 19. und 25. Stunde, KGA II/6, 38. 47. 62; DERs, Kirchengeschichte 1825/26, KGA II/6, 127; 
folgt schon daraus, daß Judentum und hellenisches Heidentum eben die beiden geistigen Mächte der Welt sind, in die das Christentum cintrat, auf die es wirkte, in der es Gestalt annahm und in der es sich behaupten mußte. ${ }^{52}$

DERs., Kollelstaneum 20, KGA II/6, 147. Die häresiologische Windrose der Glaubenslehre sieht die ebionitisch-pelagianische Ketzerei als mehr judaisierend, die doketisch-manichäisch als hellenisierend an (Schleiermacher, Der christliche Glaube ${ }^{2}$, 22, KGA I/13,1, 155-160).

52 Schleiermacher kann dem Ebionitismus auch die polytheistische Deutung der Göttlichkeit Christi als ethnisierende Ketzerei gegenüberstellen (Schleizrmacher, Kollektaneum 45, KGA II/6, 152; vgl, auch DERs., Notizen zur Trinitätslehre, in: KGA II/6, 757f. 765; DERs., Über den Gegensaz zwischen der Sabellianischen und der Athanasianischen Vorstellung von der Trinität, in: Theologische Zeitschrift 3 [1822], 295-408, hier 296-301, in: KGA I/10, hg.v. Hans-Friedrich Traulsen/Martin OHsт, Berlin/New York 1990, 226-228) und andererseits zugeben, daß der Gnostizismus nicht nur im hellenischen Heidentum, sondern auch im Judentum verwurzelt ist (SCHLEIERMACHer, Kirchengeschichte 1821/22, 18. Stunde, KGA II/6, 46), - Bei manchen Phänomenen ist Schleiermacher sich auch nicht sicher, ob er sie mehr auf der jüdischen oder der heidnischen Seite einordnen soll: Den von Euseb gelegentlich benutzten frühchristlichen Schriftsteller Hegesipp ordnet er zunächst unter den häretischen Judenchristen ein, d.h. unter denen, die die Einzigartigkeit Christi und die Neuheit des Christentums gegenüber dem Alten Testament nicht voll crfaßt hätten (Schleiermacher, Kirchengeschichte 1821/22, 14. Stunde, KGA II/6, 36; Ders., Kollektaneum 25, KGA II/6, 147f.). Das zweite kirchengeschichtliche Kompendium macht Hegesipp dann anhand eines nicht bei Euseb, sondern in der Bibliothek des Photius überlieferten Fragments vom Judaisten zu einem Gegner des Chiliasmus und einer unreflektierten und unkritischen Rezeption des Alten Testaments in der christlichen Kirche (SchleIERMAcher, Kirchengeschichte 1825/26, KGA II/6, 131. 716f.). Tatsächlich gibt die Stelle (aus den Quaestiones disputatae des Monophysiten und Tritheisten Stephan Gobar bei Photrus, Bibliotheca codex 232, in: Ders., Bibliothèque, Bd. 5, hg. y. René Henry, Paris 1967, 70) dies aber gar nicht her: Hegesipp argumentiert dort mit Matth 13, 16 gegen Jes64,3; 1 Kor 2,9 dafür, daß die Heilsgüter nicht unsichtbar und unhörbar, sondern sichtbar und hörbar seien. Nach Schleiermacher berief Hegesipp sich aber gegen den Chiliasmus auf Joh 20,29. - Die schroffe, asketische Abwertung der Materie bei den auf Tatian den Assyrer zurlickgehenden Enkratiten wiederum hält Schleiermacher zunächst für übertriebenen Antijudaismus (ScHLEIermacher, Kollektaneum 27, KGA II/6, 148). Dann jedoch kommt er zur genau entgegengesetzten Einordnung des Phänomens: Tatians Strenge sei eine »modificirte jüdische Ansicht«. Die Verteufelung der Materie finde sich zwar auch im Gnostizismus, erkläre sich aber bei Tatian daraus, daß der Satan nach alttestamentlich-jüdischer Lehre die Menschen mit der Materie betrogen und zu Fall gebracht habe. Fleisch und Alkohol, die Tatian verbot, übten von allen Nahrungsmitteln die stärkste Gewalt auf den Menschen aus, und das Fleisch lasse sich darüber hinaus nicht genau vom Blut trennen, in dem nach juidischer Vorstellung die Seele als Prinzip der Lebendigkeit lokalisiert wird. Das enkratitische Eheverbot erscheine bei dem großen Wert, den das Judentum auf Nachkommenschaft legt, zwar als ganz unjüdisch, doch auch dies lasse sich mit einer judaisierenden Tendenz vereinbaren, denn das Interesse an der Vermehrung des Volkes durch Fortpflanzung gehöre nur zur nationalen Seite des Judentums, nicht zur eigentlichen Religion der Juden (Schleisrmacher, Kirchengeschichte 1821/22, 21. Stunde, KGA II/6, 51f.). Offenbar hält Schleiermacher Gesetzlichkeit in ätßerlichen Dingen für etwas genuin Jüdisches, dem hellenisch-gnostischen Prinzip der äußerlichen Beliebigkeit Entgegenstehendes. Im zweiten Kompendium revidiert Schleiermacher diese Deutung wieder: Tatians Feindschaft gegen die Materie sei gnostisch und stelle einen Gegensatz gegen das Judaisierende dar (Schleigrmacher, Glosse zu Kirchengeschichte 1821/22, 21. Stunde, KGA II/6, 51 Fußtext; DERS., Kirchengeschichte 1825/26, KGA II/6, 132, und SW I/11, 132f.; DERs., Kollektaneum 960, KGA II/6, 387). 
Über die Gnosis hat Schleiermacher eigene Quellenforschungen angestellt ${ }^{53}$ und zu dem Problem, wie die verschiedenen gnostischen Gruppen und Phänomene sinnvoll erfaßt und einander zugeordnet werden können, eigenständige Lösungsvorschläge beigesteuert. ${ }^{54}$ Er betrachtet den Gnostizismus als ein Ineinander von wissenschaftlichphilosophischen, mythologischen und religiösen Elementen: Spekulative Kosmologie, Dualismus zwischen Geist und Materie, Doketismus, Theodizee und Soteriologie vereinigen sich zu einer philosophisch-theologischen Weltanschauung, in der die Schöpfung der materiellen Welt und die Erlösung in genauem Gegensatz gegeneinander stehen.

Die beginnende rechtgläubige Theologie seit etwa 200 antwortet auf diese Extravaganzen mit zwei Dogmen: der göttlichen Monarchie und der Logos-Christologie. Das erste richtet sich besonders gegen die Gnosis: Es setzt der Abwertung der Schöpfung und des Alten Testaments entgegen, daß alle göttlichen Offenbarungen und Heilsveranstaltungen aus einer gemeinsamen Quelle kämen. Gegen die Ebioniten ist vor allem das zweite Dogma gerichtet, die Logos-Christologie. Sie wehrt die unbeschränkte Gleichsetzung des Erlösers mit den zu Erlösenden ab, die Herabsetzung Christi zu einem bloßen Menschen (dafür war der Begriff des Logos geeigneter als der noch weniger eindeutige Begriff des Gottessohns). Die dogmatische Literatur bis zu Origenes beruht auf eben diesen beiden Sätzen. ${ }^{55}$

Am Ende der Periode sind die beiden Sätze christliches Allgemeingut. Das Christentum ist sich seiner Besonderheit gegenüber dem Judentum, der heidnischen Mythologie und der hellenischen Metaphysik bewußt geworden, und so kommen Vermischungen mit diesen geistigen Größen von nun an nicht mehr vor: ${ }^{56}$

\section{6.}

Hat damit das Judenchristentum aufgehört? Nicht ganz. Es lebt fort, im Katholizismus. Damit ist hier nicht die Idee der Papstmonarchie gemeint, sondern das Bewußtsein der Einheit der Kirche mit sich selbst, wie es im zweiten Jahrhundert allmählich hervordringt. ${ }^{57}$ Die Idee des Katholischen meint nicht nur die Einheit der Kirche im Raum,

53 Schleiermacher, Kollelktaneen 23f; 30; 49-54; 67; 76-87; 107; 110-139; 163, KGA II/6, 147. 149. 153. 156. 158-160. 163-172. 177.

54 SchLEIERmacher, Kirchengeschichte 1821/22, 18. Stunde, KGA II/6, 46.

55 Schleirrmacher, Kirchengeschichte 1821/22, 19., 25. und 32. Stunde, KGA II/6, 47f. 62. 79.

56 SchlerzRmacher, Kirchengeschichte 1821/22, 6. Stunde, KGA II/6, 25. 484; DERs., Kirchengeschichte 1825/26, KGA II/6, 694f. - In der kirchlichen Statistik indessen deutet Schleiermacher das Christentum der Abessinier als ein erstarrtes, vom Judentum innerlich noch nicht richtig abgelöstes Christentum; Indiz dafür sind ihm die zahlreichen jüdischen Gebräuche in dieser Kirche: Beschneidung, Reinheits- und Reinigungsgesetze, Vielehe der Herrscher und das Aufstellen einer Nachbildung der Bundcslade im sakralen Raum; vgl. ScinLenrmmacrme, Kirchliche Statistik 1827, 8. Stunde, KGA II/16, 216f; DERs., Kirchliche Statistik 1833/34, 8. Stunde, KGA II/16, 485f.; DERs., Semitischer Zweig 6; 13; 17, in: KGA IL/16, 20. 24. 26.

57 SCHLEIERMACHER, Kirchengeschichte 1825/26, KGA II/6, 717. 
die Ökumene, sondern auch in der Zeit. ${ }^{58}$ Daher rechnet der Katholizismus nicht damit, daß sich die Kirche im Laufe ihrer Geschichte fortentwickle. ${ }^{59}$ Und umgekehrt dehnt er die Einheit der Kirche auch in die Vergangenheit aus, in die Vorgeschichte des Christentums, und hält Altes und Neues Testament ohne Unterschied für eins. Auch in die evangelischen Bekenntnisschriften ist diese Vorstellung eingegangen, nämlich als Lehre von der wahren Kirche von Anbeginn der Menschheit an. ${ }^{60} \mathrm{Im}$ Unterschied zum Ebionitismus wird hier freilich nicht Christus ins Alte Testament eingegliedert, sondern umgekehrt das Alte Testament in die Kirchengeschichte.

Wenn Cerdo, Marcion und später die Manichäer demgegenüber die Einheit und Einerleiheit der Heilsgeschichte bestreiten, so ist das für Schleiermacher angesichts ihrer Weltverneinung zwar nicht die volle Wahrheit, aber doch auch wieder eine verständliche, sogar sympathische Reaktion gegen eine unkritische Rezeption des Alten Testaments, ein Wachhalten des Bewußtsein, daß das Christentum als etwas Neues in die Welt kommt. ${ }^{61}$ So versucht Schleiermacher, zwischen beiden Einseitigkeiten, der monistisch-katholischen und der dualistisch-gnostischen, zu vermitteln: Cerdo als gemäßigter Dualist und etwa Justin der Märtyrer als Vertreter des frühen Katholizismus, beide wirksam in der Mitte des zweiten Jahrhunderts, hätten sich wohl darauf verständigen können, daß die Vorstellungen des Neuen Testaments im Alten latitierten, also schon vorhanden seien, aber eben nur verborgen, so daß man sowohl von heilsgeschichtlicher Kontinuität sprechen könne als auch von Umbruch und Neuanfang; doch dazu fehlte es an der nötigen Kommunikation zwischen Cerdos Heimat Syrien und Justins Heimat Samarien. ${ }^{62}$ Und in der Glaubenslehre läßt Schleiermacher die Annahme »einer einzigen Kirche Gottes von Anbeginn des Menschengeschlechtes bis zum Ende desselben« insoweit gelten, als sie nicht die Eigenständigkeit des Christentums gegenüber der Religion des Alten Testaments nivelliert, sondern ausspricht, daß die Wirksamkeit Christi und die Kirche als ihr Gegenstand keine Teilung leiden, weder durch nationale

58 Vgl. den klassischen Ausspruch des Vinzenz von Lerinum, Commonitorium 2,3 (hg.v. Reginald Stewart Moxon, Cambridge 1915, 10): Wir müssen das als katholisch bewahren, was überall, immer und von allen geglaubt wurde.

59 Vgl. Schleigrmacher, Christliche Sitte 1822/23, SW I/12, 72, 123. 384.

60 Schleiermacher, Der christliche Glaube ${ }^{2}, \$ 156,1$, KGA I/13,2, 450-453. Schleiermacher hält diese Lehre ausdrücklich nicht für ein reformiert-föderaltheologisches Proprium.

61 Schleiermacher, Kirchengeschichte 1821/22, 16. und 18. Stunde, KGA II/6, 39. 45f;; Ders., Kirchengeschichte 1825/26, KGA II/6, 131. 716f, und SW I/11, 286; DERs., Kollektaneen 24; 30; 115f.; 1052, KGA II/6, 147. 149. 166. 408; DERs., Einleitung ins Neue Testament 1831/32, \$18, in: SW I/8, 64. Auch der Monarchianer Sabell wird gelobt, weil er die Theophanien des Alten Testaments nicht einfach mit dem in Christus fleischgewordenen Logos identifiziert wie die Athanasianer; Schleiermacher, Über den Gegensaz (s.o. Anm. 52), 399f, KGA I/10, 299 f.

62 Schledermacher, Kirchengeschichte 1825/26, KGA IL/6, 717. Vgl. Ders., Christliche Sitte 1826/27, SW I/12, 292f: "Es ist ein Schwanken in der Theologie zwischen der Auffassung des Christenthums als eines ganz eigenthümlichen und zwischen der Auffassung desselben als einer reinen Entwikkelung der alttestamentlichen Offenbarung. Keines von beiden kann ganz geleugnet werden [...]. Das von der Person Christi ausgehende eigenthümliche ist aber das wesentliche, alles übrige nur conditio sine qua non." 
oder kulturelle Schranken noch durch die Zeiten. Christus und sein Werk beziehen sich unbeschränkt auf alles Menschliche, auch auf die vorchristliche Vergangenheit. ${ }^{63}$

Und so kann Schleiermacher zustimmend den frühchristlichen Theologen Clemens von Alexandrien zitieren:

"Gott hat den Juden das Gesez gegeben und den Griechen die Philosophie. Aus beiden werden Christen. ${ }^{64}$

63 Schletermacher, Der christliche Glaube ${ }^{2}, \$ 12,3$, KGA I/13,1, 105f; vgl. $\$ 156,3$, KGA I/13,2, 454f. Vgl. BeciKmanN, aaO. (s.o. Anm. 30), 70f.

64 Schleibrmacher, Kollektaneum 48, KGA II/6, 152, nach Clemens von Alexandrien, Stromata VI, 5, $\$ 41,6-42,3$, in: GCS 52 (Clemens II), hg.v. Otto STÄHLIN/Ludwig Früchtel/Ursula Treu, Berlin-Ost ${ }^{4} 1985,452$; vgl. Schleiermacher, Kirchengeschichte 1821/22, 16. Stunde, KGA II/6, 39 Fußtext; DERs., Kirchengeschichte 1825/26, SW I/11, 160. Vgl. auch Ders., Der christiche Glaube $^{2}, \$ 12,3$, KGA I/13,1, 105 (hier wird Clemens, Stromata VI, 17, $\$ 159,9$, in: GCS 524, 514, zitiert); DERs., Theologische Enzyklopädie 1831/32, $\$ 46$ (s.o. Anm. 12), 53. 